\title{
A VLBI study of GHz-Peaked-Spectrum radio sources
}

\author{
II. Additional VLBI images at $6 \mathrm{~cm}$ \\ C. Stanghellini ${ }^{1,4}$, C.P. O'Dea ${ }^{2}$, and D.W. Murphy ${ }^{3}$ \\ 1 Istituto di Radioastronomia del CNR, C.P. 141, I-96017 Noto SR, Italy \\ 2 Space Telescope Science Institute, 3700 San Martin Drive, Baltimore, MD 21218, U.S.A. \\ 3 Jet Propulsion Laboratory, MS 238-600, 4800 Oak Grove Drive, Pasadena, CA 91109, U.S.A. \\ ${ }^{4}$ Visitor at the Space Telescope Science Institute, Baltimore
}

Received July 15; accepted August 21, 1998

\begin{abstract}
We present VLBI observations at $5 \mathrm{GHz}$ of $11 \mathrm{GHz}$ Peaked Spectrum (GPS) or candidate GPS sources. Two of them belong to the complete sample defined by Stanghellini et al. (1998) (the GPS 1 Jy sample), while the others are selected from a heterogeneous list compiled by O'Dea et al. (1991). Morphologies of the sources presented here are similar to those found in Stanghellini et al. (1997) and in the small Compact Steep Spectrum (CSS) sources studied by Dallacasa et al. (1995).

Our results strengthen the evidence that GPS quasars tend to have core-jet or complex morphology and GPS galaxies tend to be compact symmetric objects (CSO).
\end{abstract}

Key words: galaxies: active — quasars: general — radio continuum: galaxies

\section{Introduction}

GPS radio sources have small sizes $(<500 \mathrm{pc})$ high radio luminosity $\left(>10^{26} \mathrm{~W} \mathrm{~Hz}^{-1}\right)$ and are characterized by a convex radio spectrum peaking around $1 \mathrm{GHz}$. The nature of these radio sources hosted by galaxies and quasars is still debated. They could be the early stages of powerful extended radio sources or they could be indefinitely confined to their small sizes by a high density medium which prevents the expansion of the jets and lobes outside the central region of the galaxy hosting the radio source. A review of the properties of these sources is given by O'Dea (1998).

Due to the small sizes of GPS radio sources, radio observations at the milliarcsecond scale with VLBI techniques are necessary to reveal their morphology. The ra-

Send offprint requests to: C. Stanghellini dio morphology is a critical piece of information necessary to our understanding of the nature of these sources (e.g., Wilkinson et al. 1994). The sidedness - i.e., to what extent the sources are two sided (CSO) or one sided (corejet) - constrains the effects of Doppler boosting and is related to the Lorentz factor and orientation of the jets. The morphology (i.e., Fanaroff and Riley class I or II) provides clues to the transport of energy in the jets, e.g., transonic and non-relativistic or supersonic and relativistic, respectively. The extent to which the two sides of the source are similar/symmetric also constrains the interaction of the jet with the environment, i.e., does the jet remain well collimated or it is disrupted through interaction with dense gas? The morphology also provides constraints on how the sources may evolve (e.g., Readhead et al. 1996a,b; O'Dea $\&$ Baum 1997). In order to achieve this goal we have undertaken a VLBI study to observe the objects of the GPS 1 Jy sample (Stanghellini et al. 1998) and several of the GPS candidates listed by O'Dea et al. (1991)

Stanghellini et al. (1997) present global VLBI $6 \mathrm{~cm}$ images of 9 radio sources of the GPS 1 Jy sample. Here we add 11 radio sources from the list of O'Dea et al. (1991). Two of these objects are also included in the complete sample. Most of the sources presented here have never been previously observed before at this frequency.

In following papers in this series, images at other frequencies and a complete discussion of the results of our study will be given.

$H_{0}=100 \mathrm{~km} \mathrm{~s}^{-1} \mathrm{Mpc}^{-1}$, and $q_{0}=0.5$ have been assumed throughout this paper.

\section{Observations and results}

The observations were carried out on 24 September 1989 with a global network of 11 antennas (Table 1) using the Mark 2 recording system (Clark 1973). 
V vs U for $1543+005$

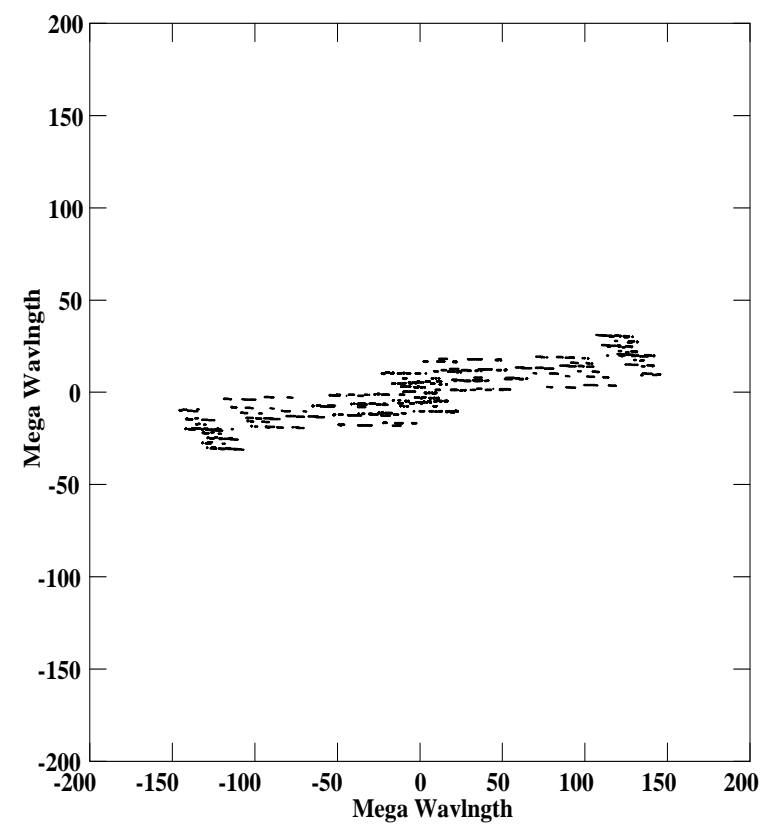

Fig. 1. UV coverage for $1543+005$

Table 1. Antennas involved in the VLBI experiment. The nominal system equivalent flux density (SEFD) in Col. 3 is given by the ratio between the system temperature (in $\mathrm{K}$ ) and the antenna gain (in $\mathrm{K} / \mathrm{Jy}$ ). The VLA was available as a phased array for a fraction of time (equivalent values in parentheses) and with a single antenna otherwise

\begin{tabular}{lrr}
\hline Antenna & $\begin{array}{r}\text { Diam. } \\
(\mathrm{m})\end{array}$ & $\begin{array}{r}\text { nominal SEFD } \\
(\mathrm{Jy})\end{array}$ \\
\hline Onsala (Swe) & 20 & 990 \\
WSRT (NL) & 93 & 107 \\
Effelsberg (Ger) & 100 & 44 \\
JBNK2 (UK) & 25 & 344 \\
Medicina (Ita) & 32 & 290 \\
HSTK (US) & 37 & 975 \\
Green Bank (US) & 43 & 130 \\
Iowa (US) & 18 & 800 \\
FDVS (US) & 26 & 650 \\
VLA (US) & $25(130)$ & $330(20)$ \\
OVRO (US) & 40 & 225 \\
\hline
\end{tabular}

The data have been calibrated and imaged in AIPS using standard procedures (for details see Stanghellini et al. 1997). The global array used for these observations spans a narrow range in latitude, thus the sources at low declination have a very elongated beam. In Figs. 1, 2, 3 we show the UV coverage for sources at declinations of 0,23 , and 60 degrees.

The list of the observed radio sources with basic information is shown in Table 2. When the redshift was not directly available we estimated it using the magnitude-
V vs U for $0039+230$

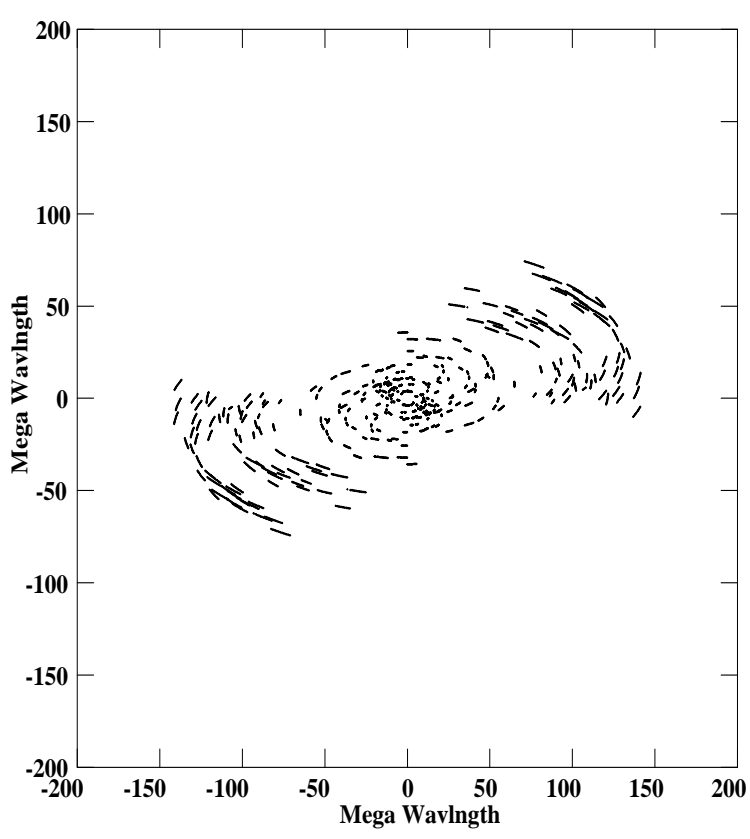

Fig. 2. UV coverage for $0039+230$

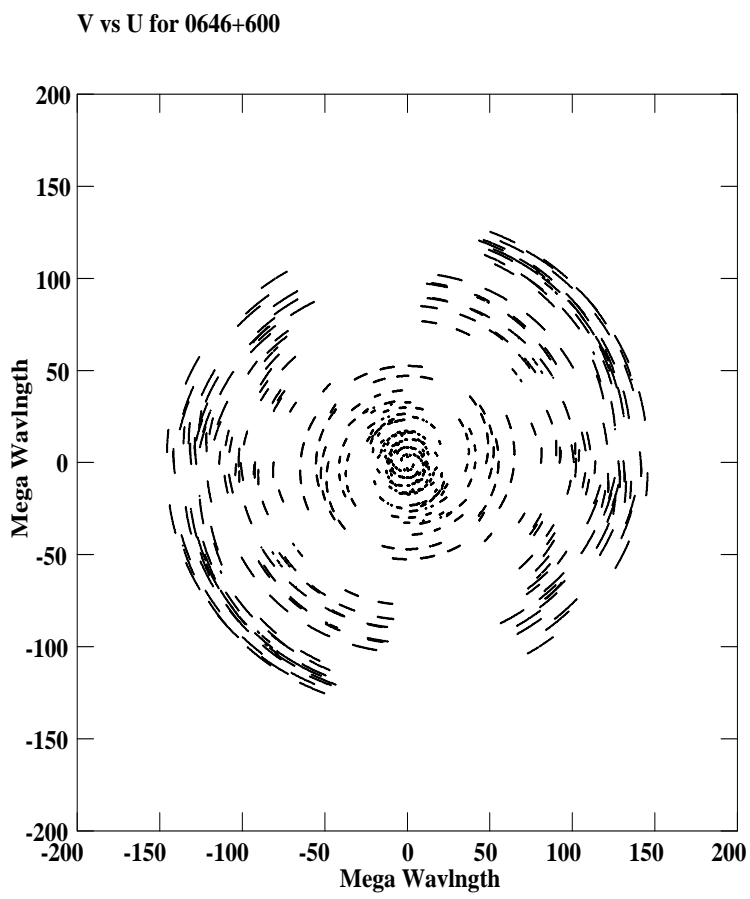

Fig. 3. UV coverage for $0646+600$

redshift relation found by O'Dea et al. (1996). The estimated redshifts are marked with an asterisk.

The parameters of the various components are shown in Table 3 as measured on the images. We report the Full Width Half Maximum (FWHM) size derived from the Gaussian fits (task IMFIT in AIPS). An asterisk following the parameter indicates that the fit gave a very 
Table 2. GPS sources. Columns 1 through 9 provide: name, optical identification, optical magnitude, redshift, linear scale factor $\mathrm{pc} / \mathrm{mas}\left[H_{0}=100 \mathrm{~km} \mathrm{~s}^{-1}\right.$ and $q_{0}=0.5$ have been assumed], maximum VLBI angular size, maximum VLBI linear size, flux density at $5 \mathrm{GHz}$ as measured from our VLBI images, references for the information given here: 1) O'Dea et al. 1991; 2) Hewitt \& Burbidge 1993; 3) Stickel \& Kühr 1993; 4) Meisenheimer \& Röser 1983; 5) Stanghellini et al. 1993; 6) O’Dea et al. 1990b; 7) O'Dea et al. in preparation; 8) Stickel \& Kühr 1992; 9) de Vries et al. 1995)

\begin{tabular}{llllccccc}
\hline \hline Source & id & $m$ & $z$ & $\begin{array}{c}\text { scale } \\
\mathrm{pc} / \mathrm{mas}\end{array}$ & $\begin{array}{c}\theta_{\max } \\
\operatorname{mas}\end{array}$ & $\begin{array}{r}l_{\max } \\
\mathrm{pc}\end{array}$ & $\begin{array}{c}S_{\mathrm{vlbi}} \\
\mathrm{Jy}\end{array}$ & ref. \\
\hline $0039+230$ & $\mathrm{EF}$ & - & - & - & - & - & 1.192 & \\
$0457+024$ & $\mathrm{Q}$ & $19.4 \mathrm{~V}$ & 2.384 & 3.9 & 15 & 59 & 1.524 & 2 \\
$0646+600$ & $\mathrm{Q}$ & $18.9 \mathrm{R}$ & 0.455 & 3.4 & 9 & 31 & 0.840 & 3,4 \\
$1045+019$ & $\mathrm{EF}$ & - & - & - & - & - & 0.288 & \\
$1100+223$ & $?$ & $<23 \mathrm{r}$ & $>1.2^{*}$ & - & - & - & 0.505 & 5 \\
$1543+005$ & $\mathrm{G}$ & $20.0 \mathrm{R}$ & 0.556 & 3.7 & 10 & 37 & 1.123 & 6,7 \\
$1732+094$ & $\mathrm{G}$ & $20.7 \mathrm{R}$ & $0.61^{*}$ & 3.8 & 15 & 57 & 0.773 & 6 \\
$2008-068$ & $\mathrm{G}$ & $21.3 \mathrm{R}$ & $0.75^{*}$ & 4.1 & 30 & 122 & 1.190 & 6 \\
$2015+657$ & $\mathrm{Q}$ & $19.7 \mathrm{R}$ & 2.845 & - & - & - & 0.684 & 3,6 \\
$2149+056$ & $\mathrm{G}$ & $20.4 \mathrm{R}$ & $0.56^{*}$ & 3.8 & 5 & 19 & 0.821 & 6,8 \\
$2153-119$ & $\mathrm{~S}$ & $21.7 \mathrm{i}$ & $0.88^{*}$ & 4.2 & 5 & 21 & 0.300 & 9 \\
\hline
\end{tabular}

Table 3. Size and flux density of the components in the VLBI images. The columns give: (1) source name and possible classification (CSO: Compact Symmetric Object; CJ: core-jet; CX: complex), (2) component identification, (3) and (4) major and minor axes (fitted Gaussian FWHM, a * indicates a rough estimate), (5) and (6) linear size derived from Cols. 3 and 4, (7) position angle, (8) total flux density of the component, (9) intrinsic brightness temperature neglecting relativistic effects, (10) minimum energy density, (11) equipartition magnetic field. The scale factor for each source is that given in Col. 5, Table 2

\begin{tabular}{|c|c|c|c|c|c|c|c|c|c|c|}
\hline $\begin{array}{l}\text { Source and } \\
\text { class }\end{array}$ & Comp. & $\begin{array}{c}\theta_{1} \\
\text { mas }\end{array}$ & $\begin{array}{c}\theta_{2} \\
\text { mas }\end{array}$ & $\begin{array}{r}l_{1} \\
\mathrm{pc}\end{array}$ & $\begin{array}{r}l_{2} \\
\mathrm{pc}\end{array}$ & $\mathrm{PA}$ & $\begin{array}{c}S_{5 \mathrm{GHz}} \\
\mathrm{mJy}\end{array}$ & $\begin{array}{c}T_{\mathrm{b}} \\
10^{9} \mathrm{~K}^{\circ}\end{array}$ & $\begin{array}{c}u_{\min } \\
10^{-6} \mathrm{erg} / \mathrm{cm}^{3}\end{array}$ & $\begin{array}{c}H_{\mathrm{eq}} \\
10^{-3} \mathrm{G}\end{array}$ \\
\hline $0039+230$ & $\mathrm{~A}$ & $<1$ & $<1$ & - & - & - & 905 & - & - & - \\
\hline \multirow[t]{2}{*}{ CX? } & B & $<1$ & $<1$ & - & - & - & 209 & - & - & - \\
\hline & $\mathrm{C} ?$ & $<1^{*}$ & $<1^{*}$ & - & - & - & $30^{*}$ & - & - & - \\
\hline $0457+024$ & $\mathrm{~A}$ & $<1$ & $<1$ & $<3.9$ & $<3.9$ & - & 753 & $>55$ & $>1300$ & $>120$ \\
\hline \multirow[t]{5}{*}{ CX } & B & $<2$ & $<2$ & $<7.8$ & $<7.8$ & - & 416 & $>7.6$ & $>290$ & $>55$ \\
\hline & $\mathrm{C}$ & $<3^{*}$ & $<3^{*}$ & $<11.8^{*}$ & $<11.8^{*}$ & - & $190^{*}$ & $>1.5$ & $>90$ & $>30$ \\
\hline & $\mathrm{D}$ & $4^{*}$ & $2^{*}$ & $15.7^{*}$ & $7.8^{*}$ & +110 & 85 & 0.77 & 80 & 30 \\
\hline & $\mathrm{E}$ & $<3$ & $<3$ & $<11.8$ & $<11.8$ & - & 44 & 0.23 & 30 & 20 \\
\hline & $\mathrm{F}$ & $<3$ & $<3$ & $<11.8$ & $<11.8$ & - & 28 & $>0.23$ & $>30$ & $>20$ \\
\hline $0646+600$ & $\mathrm{~A}$ & 0.74 & 0.37 & 2.6 & 1.3 & +31 & 368 & 41 & 540 & 75 \\
\hline \multirow[t]{3}{*}{ CX } & B & 0.67 & 0.39 & 2.3 & 1.3 & +47 & 432 & 48 & 600 & 80 \\
\hline & $\mathrm{C}$ & $<1^{*}$ & $<1^{*}$ & $<3.4^{*}$ & $<3.4^{*}$ & - & $6^{*}$ & $>0.19$ & $>15$ & $>13$ \\
\hline & $\mathrm{D}$ & $<2^{*}$ & $<2^{*}$ & $<6.8^{*}$ & $<6.8^{*}$ & - & $8^{*}$ & $>0.06$ & $>5$ & $>8$ \\
\hline $\begin{array}{c}1045+019 \\
\mathrm{P}\end{array}$ & & 2.5 & 1 & - & - & +170 & 285 & - & - & - \\
\hline $\begin{array}{c}1100+223 \\
\text { CJ? }\end{array}$ & & 2 & 1 & - & - & +77 & 486 & - & - & - \\
\hline $1543+005$ & $\mathrm{~A}$ & $<2$ & $<2$ & $<7.4$ & $<7.4$ & - & 781 & $>6.6$ & $>80$ & $>30$ \\
\hline \multirow[t]{2}{*}{ CX } & $\mathrm{B}$ & 3.3 & 0.9 & 12.2 & 3.3 & +34 & 310 & 3.5 & 90 & 30 \\
\hline & $\mathrm{C}$ & $5^{*}$ & $2 *$ & $18.5^{*}$ & $7.4^{*}$ & $+100^{*}$ & 85 & 0.29 & 13 & 12 \\
\hline $1732+094$ & $\mathrm{~A}$ & 1.3 & 0.9 & 5.0 & 3.4 & +98 & 480 & 14 & 200 & 50 \\
\hline $\mathrm{CSO}$ & B & 1.9 & 1.0 & 7.3 & 3.8 & +132 & 285 & 5.2 & 110 & 35 \\
\hline $2008-068$ & $\mathrm{~A}$ & 2.1 & 1.1 & 8.5 & 4.5 & +145 & 1010 & 16 & 220 & 50 \\
\hline \multirow[t]{2}{*}{ CSO } & B & $2.6^{*}$ & $0.9^{*}$ & $10.5^{*}$ & $3.6^{*}$ & $+149^{*}$ & 50 & 0.81 & 44 & 22 \\
\hline & $\mathrm{C}$ & $2.6^{*}$ & $2.6^{*}$ & $10.5^{*}$ & $10.5^{*}$ & - & 112 & 0.62 & 20 & 15 \\
\hline $\begin{array}{c}2015+657 \\
\text { P }\end{array}$ & & 0.8 & 0.4 & 3.0 & 1.5 & +103 & 676 & 175 & 5500 & 240 \\
\hline $2149+056$ & $\mathrm{~A}$ & $<1$ & $<1$ & $<3.7$ & $<3.7$ & - & 745 & $>25$ & $>260$ & $>53$ \\
\hline CJ & B & $4^{*}$ & $2^{*}$ & $14.8^{*}$ & $7.4^{*}$ & $+110^{*}$ & $70^{*}$ & $0.84^{*}$ & $25^{*}$ & $16^{*}$ \\
\hline $2153-119$ & $\mathrm{~A}$ & $<2$ & $<2$ & $<8.4$ & $<8.4$ & - & $200^{*}$ & $>2.0$ & $>50$ & $>24$ \\
\hline CJ & B & $<5$ & $<5$ & $<21$ & $<21$ & - & $100^{*}$ & $>0.16$ & $>7.2$ & $>9$ \\
\hline
\end{tabular}




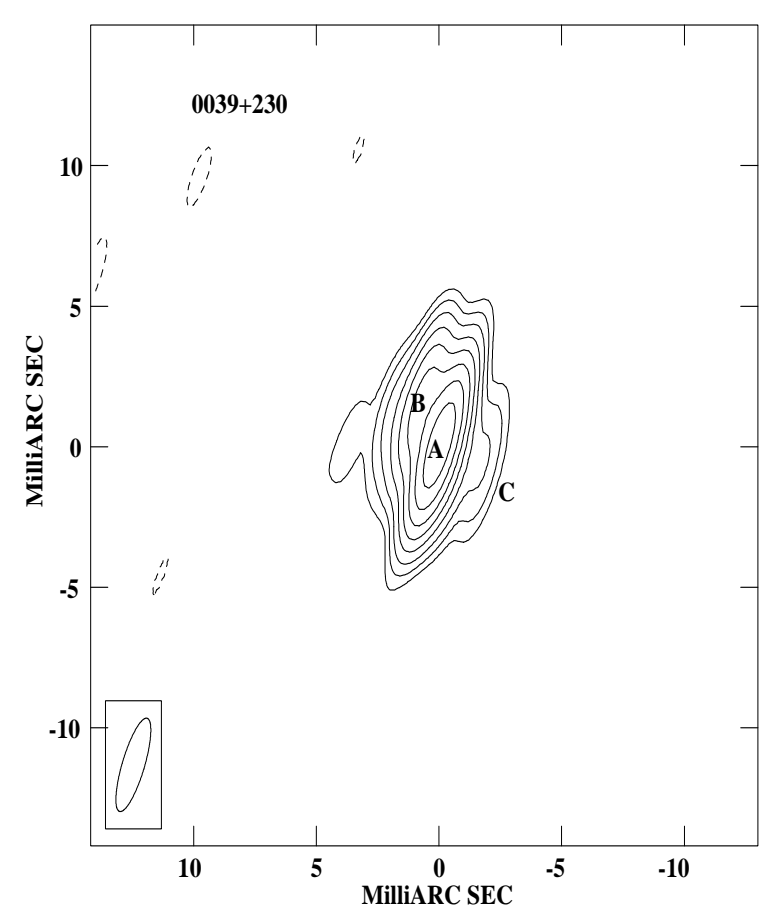

Fig. 4. 0039+230: the restoring beam is $3.5 \times 0.9$ mas in PA $-20^{\circ}$, the rms noise on the image is $1 \mathrm{mJy} /$ beam, the contour levels here and in the following figures are $-3,3,6,12,25,50$, $100,200,400,1000,2000,4000,10000$ times the rms noise, the peak flux density is $841 \mathrm{mJy} /$ beam

inaccurate value and must be considered just a rough estimate, otherwise we assume a $10 \%$ formal error.

\subsection{Comments on individual sources}

$0039+230$ : a radio spectrum of this object is shown by Spoelstra et al. (1985). Our image shows a dominant component with emission extending in the NE direction and possible emission extending also towards the west (Fig. 4).

$\mathbf{0 4 5 7 + 0 2 4}$ : this object belongs to the complete sample defined in Stanghellini et al. (1998) where the radio spectrum is presented. The VLBI image shows complex structure with 2 main blended components plus 2 or 3 weaker ones in the EW direction, and a component located NW of the main two (Fig. 5).

$\mathbf{0 6 4 6}+600$ This quasar is rather intriguing as Akujor et al. (1996) find an apparent contraction in its double radio structure. Their first epoch image at $6 \mathrm{~cm}$ is very close in time (1989.79) to the epoch of our observations (1989.73). Except for a third component which Akujor at al. claim to see which is not clearly visible in our image, our results are in agreement. The fitted sizes for the 2 main components are in agreement and we also find a separation of 3 mas between the two peaks. In Fig. 6 we show the image from our data. The weak features labeled as $\mathrm{C}$ and $\mathrm{D}$ are real (although they resemble residual errors of the hybrid mapping procedure) because they are present in

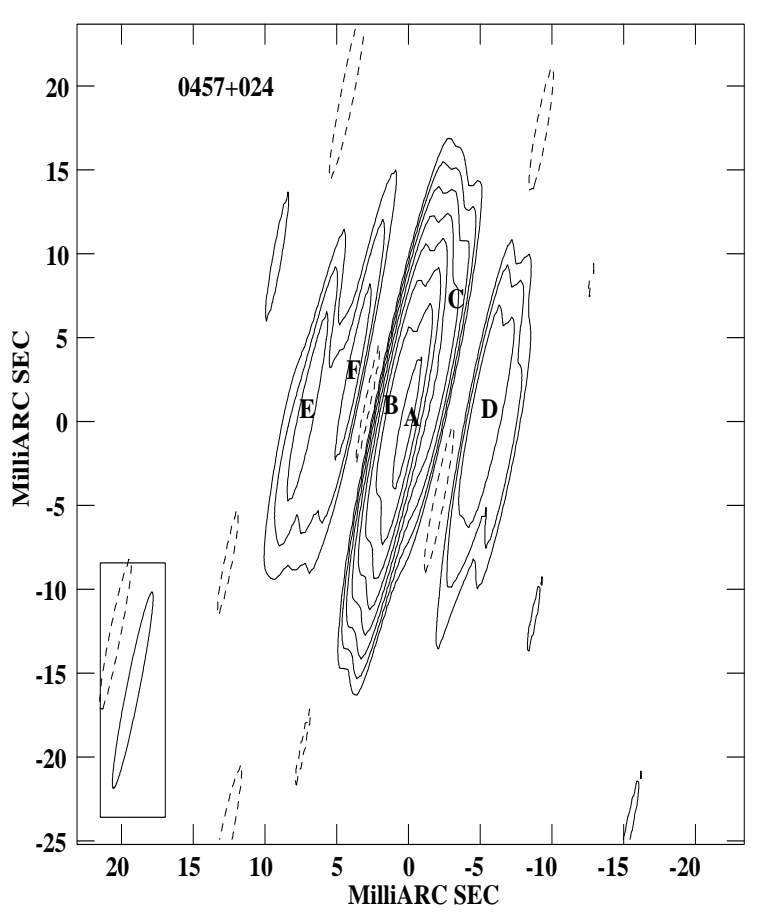

Fig. 5. $0457+024$ : the restoring beam is $12 \times 0.9$ mas in PA $-13^{\circ}$, the $\mathrm{rms}$ noise on the image is $1.3 \mathrm{mJy} /$ beam, the peak flux density is $726 \mathrm{mJy} /$ beam

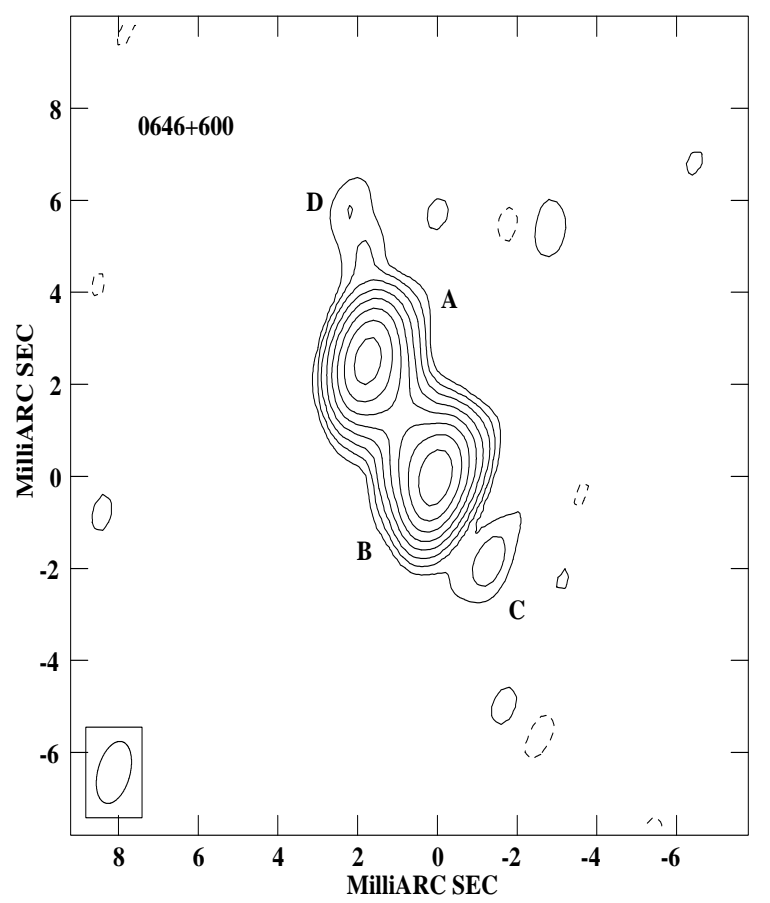

Fig. 6. $0646+600$ : the restoring beam is $1.4 \times 0.8$ mas in PA $-19^{\circ}$, the $\mathrm{rms}$ noise on the image is $0.5 \mathrm{mJy} /$ beam, the peak flux density is $338 \mathrm{mJy} /$ beam

the same position in the $5 \mathrm{GHz}$ image shown by $\mathrm{Xu}$ et al. (1995).

1045+019: the VLA radio data presented by Stanghellini et al. (1990) show a rather flat spectrum and the presence of significant extended emission, making it unlikely 


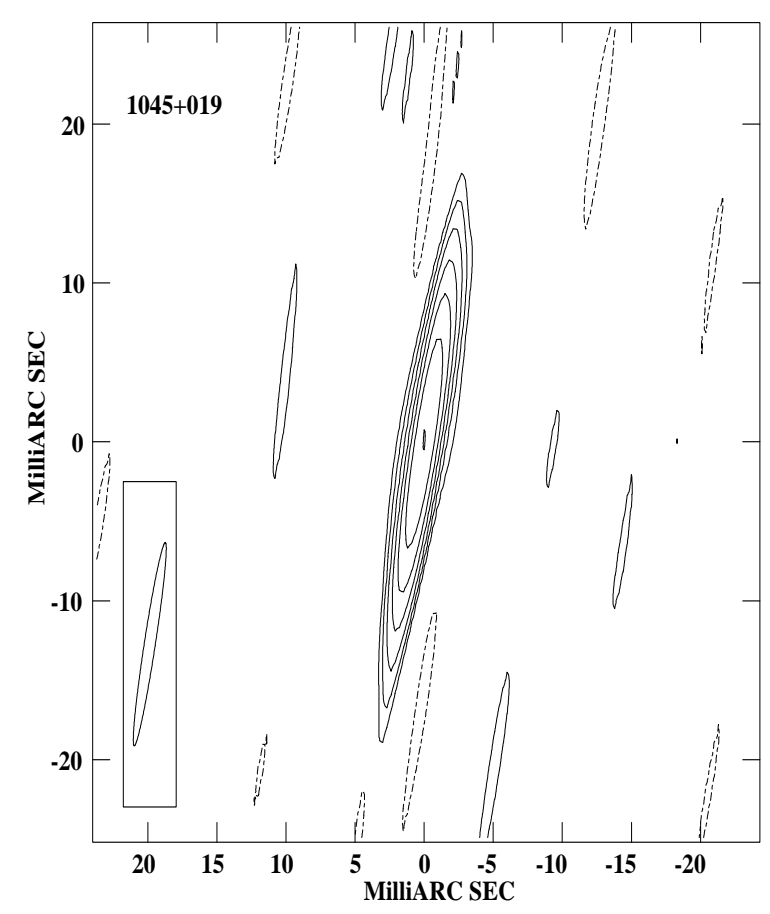

Fig. 7. 1045+019: the restoring beam is $13 \times 0.8$ mas in PA $-10^{\circ}$, the rms noise on the image is $0.8 \mathrm{mJy} /$ beam, the peak flux density is $164 \mathrm{mJy} /$ beam

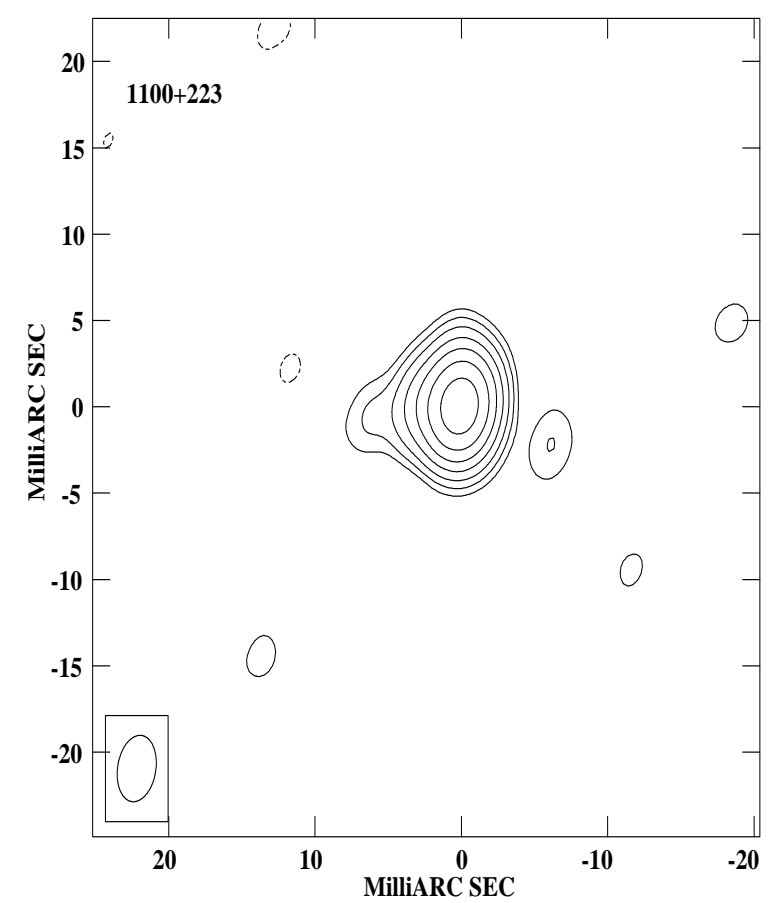

Fig. 8. $1100+223$ : the restoring beam is $3.9 \times 2.6$ mas in PA $-12^{\circ}$, the rms noise on the image is $1.2 \mathrm{mJy} /$ beam, the peak flux density is $382 \mathrm{mJy} /$ beam

that this source is really a GPS sources. The VLBI image presented here has a single component (Fig. 7).

$1100+223$ : the radio spectrum is presented by O'Dea et al. (1990a). Our VLBI image shows a single dominant component and a hint of emission towards the east (Fig. 8).

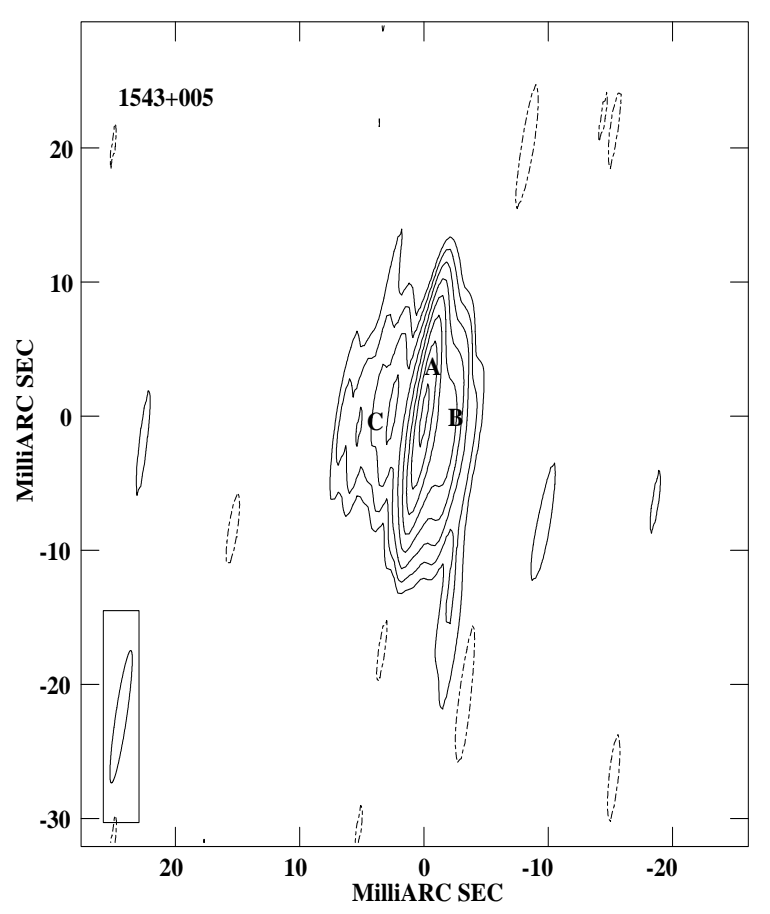

Fig. 9. $1543+005$ : the restoring beam is $10 \times 0.9$ mas in PA $-9^{\circ}$, the $\mathrm{rms}$ noise on the image is $1.3 \mathrm{mJy} /$ beam, the peak flux density is $616 \mathrm{mJy} /$ beam

1543+005: the radio spectrum presented by Stanghellini et al. (1998) does not show evidence of a clear turnover down to a frequency of $365 \mathrm{MHz}$, suggesting that this object is better classified as a CSS radio source. The VLBI image has a dominant component and emission extending to both sides (Fig. 9).

1732+094: a radio spectrum of this object is shown by Spoelstra et al. (1985). The radio image presented here shows two similar barely resolved components separated by 15 mas (Fig. 10).

2008-068: this is the second of the 11 sources presented here which belongs to the complete sample defined in Stanghellini et al. (1998). The total radio spectrum is also shown in that article. The VLBI image shows two components separated by 25 mas and a candidate core close to the strongest one (Fig. 11).

2015+657: the radio spectrum for this candidate GPS radio source is shown by Spoelstra et al. (1985). Our VLBI image shows a single component (Fig. 12). The hint of emission to the west is likely an artifact not removed by the hybrid mapping procedure. A VLBI image at $6 \mathrm{~cm}$ is also shown by Taylor et al. (1994). Their more sensitive observations reveal a core-jet morphology with a weak jet pointing from the dominant component towards the NW direction.

2149+056 The GPS radio spectrum is presented by Stanghellini et al. (1998). The VLBI image shows a possible core-jet morphology pointing towards the west (Fig. 13). 


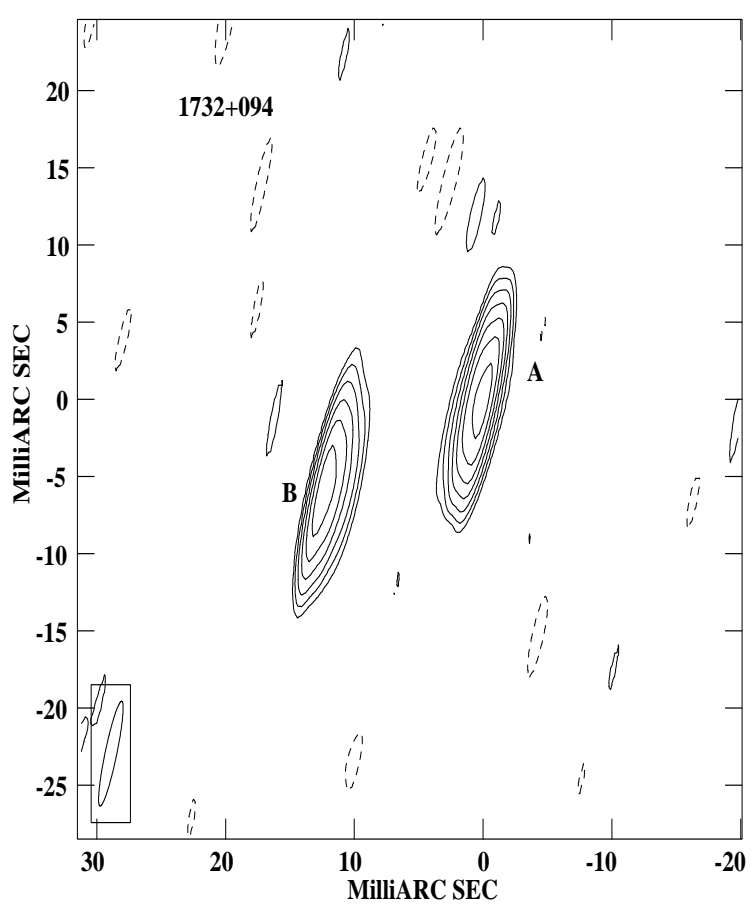

Fig. 10. $1732+094$ : the restoring beam is $7 \times 0.9$ mas in $\mathrm{PA}$ $-14^{\circ}$, the $\mathrm{rms}$ noise on the image is $1 \mathrm{mJy} /$ beam, the peak flux density is $286 \mathrm{mJy} /$ beam

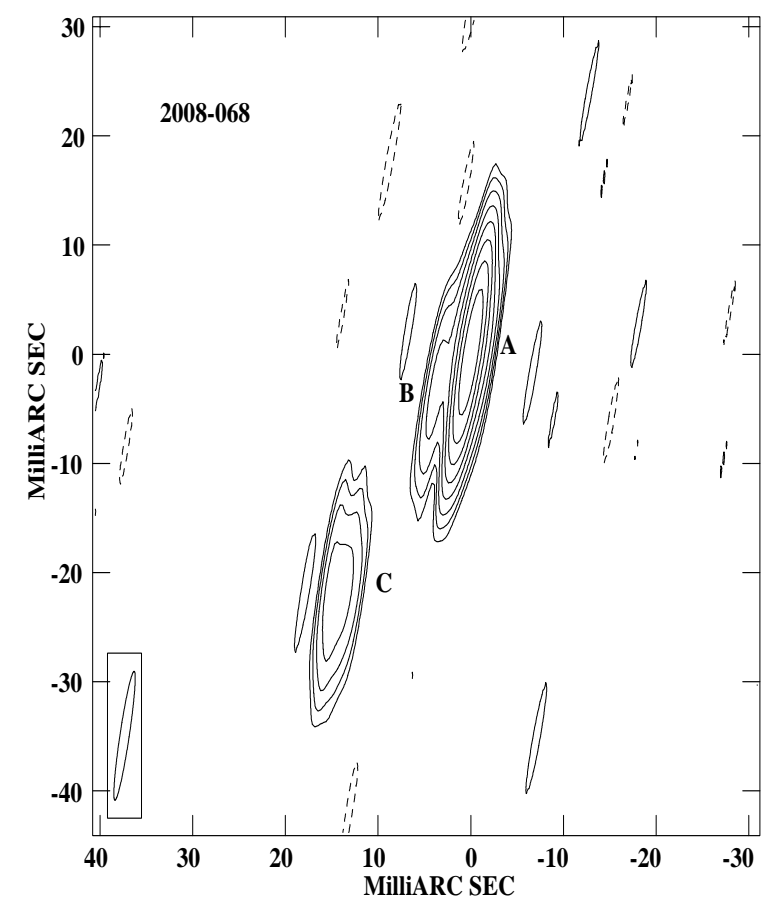

Fig. 11. 2008-068: the restoring beam is $12 \times 1$ mas in PA $-10^{\circ}$, the rms noise on the image is $1 \mathrm{mJy} /$ beam, the peak flux density is $612 \mathrm{mJy} /$ beam

2153-119: There is little information in the literature available for this source. Our VLBI image shows a core-jet

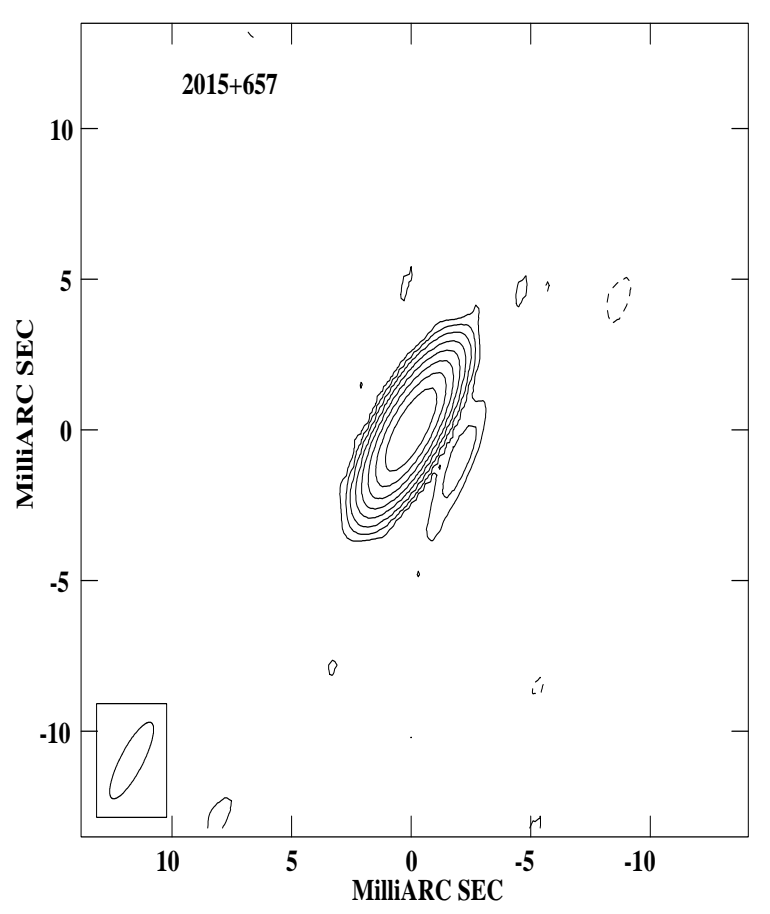

Fig. 12. 2015+657: the restoring beam is $3 \times 0.9$ mas in PA $-34^{\circ}$, the $\mathrm{rms}$ noise on the image is $0.6 \mathrm{mJy} /$ beam, the peak flux density is $545 \mathrm{mJy} /$ beam

morphology pointing in the north-east direction (Fig. 14).

The sources observed here exhibit the same morphologies already seen in GPS radio sources, i.e., some have a compact symmetric morphology while others have a corejet morphology.

Stanghellini et al. (1996) on the basis of the morphological information available in literature for the GPS 1 Jy sample find that GPS quasars have preferentially corejet or complex morphology and GPS galaxies tend to be CSO. The objects presented here (excluding 1045+019 and $1543+005$ which are not genuine GPS sources) reinforce this evidence as all the quasars and the stellar object have complex or core-jet morphology and 2 of the 3 GPS galaxies seem to have a symmetric structure. In Fig. 15 we update the histogram of the morphology versus the optical identification presented by Stanghellini et al. (1996) with the new morphological classifications proposed here for 2 objects of the complete sample.

The morphological differences observed between GPS galaxies and quasar may be interpreted in terms of a unification scheme where GPS quasars are the GPS galaxies seen along the jet axis. A problem for this interpretation is the very different redshift distributions for GPS galaxies and quasars (O'Dea et al. 1991; Stanghellini et al. 1998; Snellen 1997). Thus, the relationship between GPS galaxies and quasars needs to be studied further, taking into consideration radio variability, polarization, spectral shape (cf. Snellen et al. 1998), etc., which are the 


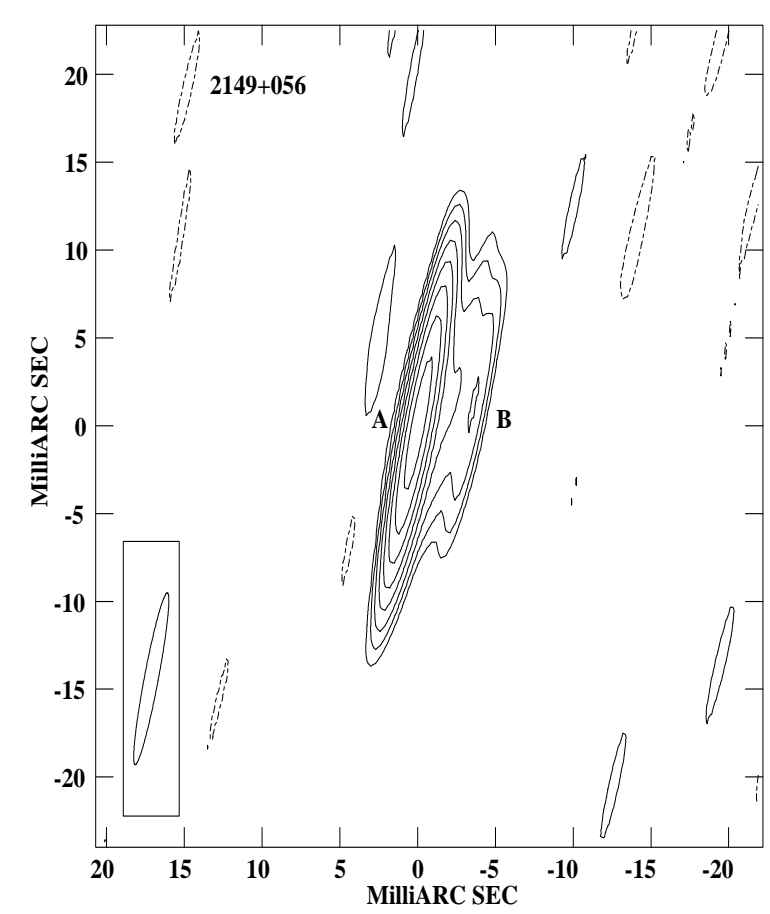

Fig. 13. $2149+056$ : the restoring beam is $10 \times 0.9$ mas in PA $-12^{\circ}$, the $\mathrm{rms}$ noise on the image is $1 \mathrm{mJy} /$ beam, the peak flux density is $618 \mathrm{mJy} /$ beam

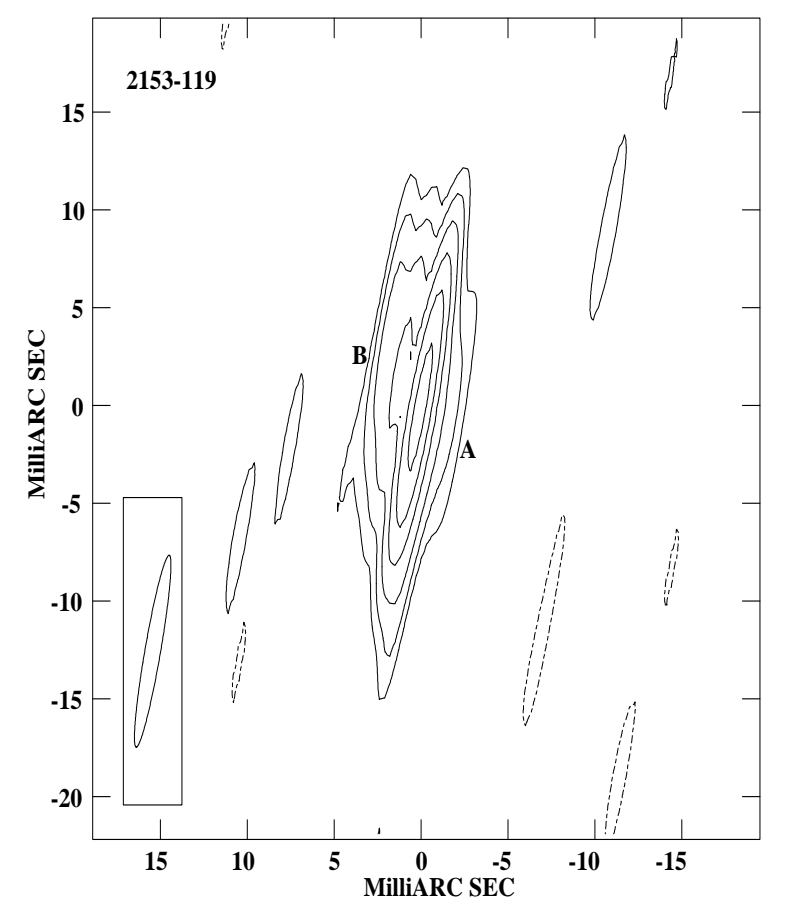

Fig. 14. 2153-119: the restoring beam is $10 \times 0.9$ mas in PA $-11^{\circ}$, the rms noise on the image is $1.2 \mathrm{mJy} /$ beam, the peak flux density is $163 \mathrm{mJy} /$ beam

topics of another study in this series of papers (Stanghellini et al. 1998). The strong link between GPS galaxy identification and CSO radio structure indicates

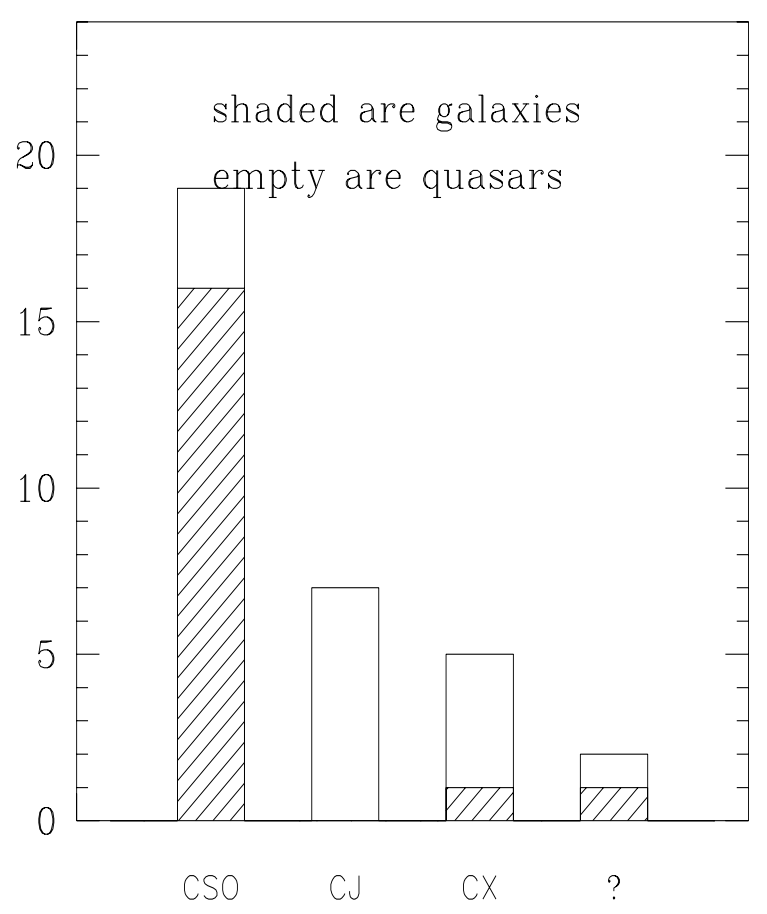

Fig. 15. Morphology versus identification for the GPS 1 Jy sample. This is an update of the corresponding histogram shown in Stanghellini et al. (1996). (CSO:Compact Symmetric Object; CJ: core-jet; CX: complex; ?: unknown)

that symmetric radio structure is a fundamental property of the GPS radio galaxies.

We note that most of the morphological classifications proposed here are tentative and based on their appearance in a VLBI image at a single frequency. However, we are not able to reliably classify a source as having structure on both sides of the core until we can locate the core with some confidence. Observations at another frequency can provide the spectral information which is necessary to identify the different components, possibly locate the core, and then confirm our classifications for the resolved sources (see e.g., Taylor et al. 1996). Higher resolution observations are instead necessary to reveal more details in the unresolved or barely resolved objects.

Acknowledgements. C.S. wishes to thank the STScI Collaborative Visitor Program for providing support for his visits. We have made use of the NASA/IPAC Extragalactic Database, operated by the Jet Propulsion Laboratory, California Institute of Technology, under contract with NASA.

\section{References}

Akujor C.E., Porcas R.W., Smoker J.V., 1996, A\&A 306, 391 Clark B.G., 1973, Proc. IEEE 61, 1242

Dallacasa D., Fanti C., Fanti R., Schilizzi R.T., Spencer R.E., 1995, A\&A 295, 27

Hewitt A., Burbidge G., 1993, ApJS 87, 451

Meisenheimer K., Röser H.-J., 1983, A\&AS 51, 41

O'Dea C., 1998, PASP 110, 493 
O’Dea C.P., Baum S.A., 1997, AJ 113, 148

O'Dea C.P., Baum S.A., Stanghellini C., Morris G.B., Patnaik A., Gopal-Krishna, 1990a, A\&AS 84, 549

O'Dea C. P., Baum S.A, Morris G.B., 1990b, A\&AS 82, 261 O'Dea C.P., Baum S.A., Stanghellini C., 1991, ApJ 380, 66

O'Dea C.P., Stanghellini C., Baum S.A., Charlot S., 1996, ApJ 470, 806

Readhead A.C.S., Taylor G.B., Xu W., Pearson T.J., Wilkinson P.N., Polatidis A.G., 1996a, ApJ 460, 612

Readhead A.C.S., Taylor G.B., Pearson T.J., Wilkinson P.N., 1996b, ApJ 460, 634

Snellen I., 1997, PhD Thesis, Univ. of Leiden

Snellen I.A.G., Schilizzi R.T., De Bruyn A.G., Miley G.K., 1998, A\&A 333, 70

Spoelstra T.A.T., Patnaik A.R., Gopal-Krishna, 1985, A\&A 152,38

Stanghellini C., Baum S.A., O'Dea C.P., Morris G.B., 1990, A\&A 233, 379

Stanghellini C., O’Dea C.P., Baum S.A., Laurikainen E., 1993, ApJS 88, 1

Stanghellini C., Dallacasa, O'Dea C.P., Baum S.A., Fanti R.,
Fanti C., 1996, in Proceedings of the Second Workshop on Gigahertz Peaked Spectrum and Compact Steep Spectrum Radio Sources, Snellen I.A.G., Schilizzi R.T., Röttgering H.J.A. \& Bremer M.N. (eds.), Leiden, Leiden Observatory, p. 4

Stanghellini C., O'Dea C.P., Baum S.A., Dallacasa, Fanti R., Fanti C., 1997, A\&A 325, 943

Stanghellini C., O'Dea C.P., Dallacasa, Baum S.A., Fanti R., Fanti C., 1998, A\&AS 131, 303

Stickel M., Kühr H., 1992, A\&AS 100, 395

Stickel M., Kühr H., 1993, A\&AS 101, 521

de Vries W.H., Barthel P.D., Hes R., 1995, A\&AS 114, 259

Taylor G.B., Readhead A.C.S., Pearson T.J., 1996, ApJ 463, 95

Taylor G.B., Vermeulen R.C., Pearson T.J., Readhead A.C.S., Henstock D.R., Browne I.W.A., Wilkinson P.N., 1994, ApJS 95, 345

$\mathrm{Xu}$ X., Readhead A.C.S., Pearson T.J., Polatidis A.G., Wilkinson P.N., 1995, ApJS 99, 297

Wilkinson P.N., Polatidis A.G., Readhead A.C.S., Xu W., Pearson T.J., 1994, ApJ 432, L87 